\title{
Anosmia y enfermedad por Coronavirus 2019 (COVID-19): ¿Qué debemos saber?
}

\author{
Anosmia and coronavirus disease 2019 (COVID-19): ¿What should we know?
}

\author{
Valeria Sepúlveda C¹, Sofía Waissbluth A², Claudia González G².
}

\begin{abstract}
RESUMEN
Nos enfrentamos actualmente a una pandemia mundial de gran impacto, como resultado de la rápida propagación de un nuevo coronavirus, SARS-CoV-2, causante de la enfermedad COVID-19. Todavía nos falta entender la fisiopatología de esta enfermedad, y la variedad de signos y síntomas que puede generar. Se ha descrito un porcentaje no menor de pacientes que desarrollan alteraciones del olfato y del gusto. La comunidad científica y médica está constantemente publicando sus observaciones sobre este tema, un proceso dinámico con abundante información. Se realizó una revisión exhaustiva de la literatura sobre las alteraciones del olfato y del gusto en los pacientes con COVID-19. Se observó una variabilidad importante en la incidencia de hiposmia/anosmia, de 5,1\% hasta $85,7 \%$, y de alteraciones del gusto de 5,6\% hasta 88,8\%. Esto se debe a que la mayoría de los estudios son principalmente retrospectivos, y varios ocuparon encuestas sin evaluación objetiva del olfato o gusto, limitando establecer con claridad la incidencia de estas alteraciones. Se observó que las alteraciones del olfato y del gusto son en general, frecuentes en estos pacientes, y presentan un buen pronóstico.
\end{abstract}

Palabras clave: Olfato, gusto, anosmia, COVID-19, SARS-CoV-2.

\begin{abstract}
We are currently facing a global pandemic of great impact, as a result of the rapid spread of a new coronavirus, SARS-CoV-2, which causes coronavirus disease (COVID-19). We have yet to understand the pathophysiology of this disease, as well as the variety of signs and symptoms it can cause. The scientific and medical communities are constantly publishing their observations on this subject, a dynamic process with a wealth of information. An exhaustive review of the literature on smell and taste alterations in patients with COVID-19 was carried out. A significant variability was observed in the incidence of hyposmia/anosmia, from $5.1 \%$ to $85.7 \%$, and of taste alterations ranging from $5.6 \%$ to $88.8 \%$. This is due to the fact that most of the studies were mainly retrospective, and several occupied surveys without objective evaluation of smell or
\end{abstract}

1 Escuela de Medicina, Pontificia Universidad Católica de Chile, Santiago, Chile.

2 Departamento de Otorrinolaringología, Pontificia Universidad Católica de Chile, Santiago, Chile.

Los autores declaran no tener conflicto de interés. No existieron fuentes de apoyo financiero para este artículo.

Recibido el 26 de abril de 2020. Aceptado el 28 de abril de 2020. 
taste, limiting to establish clearly the incidence of these alterations. It was observed that alterations of smell and taste are in general frequent in these patients, and that they present a good prognosis.

Key words: Smell, taste, anosmia, COVID-19, SARS-CoV-2.

\section{INTRODUCCIÓN}

A fines del año 2019, en Wuhan, capital de la provincia de Hubei, China, un nuevo coronavirus, el Síndrome Respiratorio Agudo Severo Coronavirus 2 (SARS-CoV-2), fue considerado como la etiología de un número importante de neumonías. En febrero de 2020, la nueva enfermedad causada por el virus SARS-CoV-2 fue nombrado oficialmente por la Organización Mundial de la Salud (OMS) como Enfermedad por Coronavirus, COVID-19, por su sigla en inglés Coronavirus Disease 2019. Este virus es parte del orden Nidovirales y familia Coronaviridae, con un genoma de ARN de cadena simple. Los coronavirus infectan principalmente a las aves y los mamíferos, pero pueden infectar a los humanos y causar enfermedades en diversos grados, desde un resfriado común hasta un síndrome respiratorio agudo severo ${ }^{1}$. Se conocen dos coronavirus, SARS-CoV que fue descubierto en 2002, y el síndrome respiratorio de Oriente Medio (MERS-CoV) descubierto en 2012, que han sido capaces de producir enfermedades respiratorias graves con epidemias importantes. EI virus SARS-CoV contiene una glicoproteína en su envoltura que se une a su receptor celular, el receptor de la enzima convertidora de angiotensina 2 (ACE2), mientras que MERS-CoV se une a la dipeptidil peptidasa 4 (DPP4). Luego de fusionarse con la membrana celular, el genoma del ARN viral se libera en el citoplasma e inicia el proceso de replicación del genoma viral².

El alto potencial de transmisión interhumana de SARS-CoV-2 Ilevó rápidamente a una epidemia en China y la consecuente pandemia global. Al 26 de abril de 2020, se han confirmado 2.804.796 casos de COVID-19 y 193.710 muertes reportadas por la $\mathrm{OMS}^{3}$. Con mayor número de casos confirmados y fallecidos en Estados Unidos, España, Italia, Francia y el Reino Unido. Hasta el 26 de abril de 2020, hay 13.331 casos confirmados en Chile, con 189 fallecidos por esta enfermedad ${ }^{4}$.

\section{Transmisión e infectividad}

La transmisión de COVID-19 es por contacto cercano (menor a 1 metro), y gotitas, pero la transmisión aérea es probable y requiere mayores estudios $^{5,6}$. Se estima que el período de incubación promedio es de 3 a 9 días, con un rango entre 0 y 24 días. He y cols observaron muestras de hisopado faríngeas de individuos positivos a SARS-CoV-2, y hallaron que la carga viral más alta se encuentra al momento del inicio de los síntomas, y sugieren que el peak de la infectividad comenzaría antes de la manifestación de los síntomas ${ }^{7}$. Basados en análisis estadísticos, Tindale y cols sugieren que el paciente puede contagiar aproximadamente desde los 2,5 días previos a la aparición de síntomas 8 . Se estima que cerca del $44 \%$ de la transmisión ocurre previa a la aparición de síntomas. Incluso, se ha reportado hasta $18 \%$ de casos positivos para SARS-CoV-2 que permanecen asintomáticos, usualmente pacientes jóvenes, que también contagiarían el virus ${ }^{9}$.

Los síntomas tienden a resolver luego de 1015 días en la mayoría de los casos, sin embargo, la eliminación viral continúa pese a la remisión de éstos. Los casos leves y asintomáticos tienden a contagiar por 10 días (entre 8 y 15 días) posresolución de síntomas. Los casos graves continúan contagiando hasta 25 días desde la aparición inicial de síntomas ${ }^{9}$. Al ser un virus transmitido por gotitas, los otorrinolaringólogos están expuestos a riesgo de infección, dada la realización de un examen físico y exámenes endoscópicos que pueden generar aerosoles ${ }^{10}$. Su diagnóstico se realiza con la prueba de polimerasa reacción en cadena con transcriptasa reversa (RT-PCR) que tiene una especificidad cercana al $100 \%$ y una sensibilidad de $64 \%$, aproximadamente 9 .

\section{Clínica}

Los síntomas principales asociados al COVID-19 son fiebre $(82,2 \%)$, tos seca $(61,7 \%)$, fatiga $(44 \%)$ 
y disnea (41\%). También se describen anorexia, mialgias, náuseas, mareos, diarrea, cefalea, vómitos, dolor abdominal, éstos en menor medida ${ }^{911}$. Y síntomas de vía aérea alta, como odinofagia hasta en $17,4 \%$ y congestión nasal $(4,8 \%)^{12}$. La mayoría de los pacientes presentan enfermedad leve a moderada, pero hasta 19\% experimenta enfermedad severa 0 neumonía crítica $^{13}$, requiriendo soporte con ventilación mecánica y suelen cursar con falla multiorgánica 0 shock, incluso con desenlace fatal. Siordia y cols describen que hasta $41,8 \%$ de los pacientes con COVID-19 desarrolla síndrome de distrés respiratorio agudo (SDRA), y cerca del $35,8 \%, 45,3 \%$, y $18,9 \%$ de los casos de SDRA son leves, moderados y severos, respectivamente. La mayoría de los casos de COVID-19 se presenta entre los 30 y 79 años (87\%), seguido por el grupo entre los 20 y 29 años (8\%), mayores de 80 años (3\%) y por último los menores de 19 años $(2 \%)$. Las comorbilidades más prevalentes vistas en los casos de COVID-19 son: hipertensión (30,7\%), diabetes mellitus (14,3\%), enfermedad cardiovascular $(11,9 \%)$, enfermedad cerebrovascular $(6,6 \%)$, neoplasia maligna $(4,3 \%)$, enfermedad hepática crónica $(2,8 \%)$, enfermedad pulmonar crónica $(2,4 \%)$, enfermedad renal crónica $(2,1 \%)$, y VIH $(1,4 \%)^{9}$. Dentro de las complicaciones del cuadro, se encuentra el SDRA mencionado previamente, y en los casos severos de COVID-19 se ha descrito injuria miocárdica, falla cardíaca, falla renal aguda, coinfección con virus respiratorio sincicial 0 coinfección bacteriana por atípicos (Mycoplasma pneumoniae, Chlamydia pneumoniae, y Legionella pneumoniae) $)^{9}$.

Recientemente, se ha reportado que COVID-19 se asociaría a alteraciones del olfato y/o del gusto. En Corea del Sur, 30\% de los pacientes con COVID-19 desarrollaron hiposmia/anosmia, y en Alemania se ha reportado que 2 de cada 3 casos confirmados tienen anosmia ${ }^{14}$. En China e Italia, se han presentado un número significativo de pacientes con COVID-19 afectados con hiposmia/ anosmia. También se han reportado casos de anosmia aislada en pacientes con COVID-19, sin otros síntomas asociados. Se ha visto que pacientes menores de 40 años tienen mayor predisposición de desarrollar la forma de COVID-19 que se manifiesta únicamente por hiposmia/anosmia 0 alteraciones del gusto ${ }^{11}$.

\section{Sistema olfatorio}

El olfato, considerado el más primitivo de los sentidos, tiene la capacidad de relacionar, prevenir, alertar, recordar y generar distintos tipos de sensaciones. Un olor se define como una impresión especial percibida a través del sentido del olfato, que deriva de la acción de algunas sustancias químicas sobre el sistema olfatorio ${ }^{15}$. El sistema olfatorio tiene subdivisiones periféricas (neuroepitelio olfatorio y los fascículos nerviosos) y centrales (bulbo olfatorio y sus conexiones centrales) ${ }^{16}$. El neuroepitelio olfatorio, se sitúa en el techo de cada cavidad nasal, específicamente en la lámina cribiforme, y ocupa un área de aproximada de 1,5 a 2,5 $\mathrm{cm}^{2}$. Representa el órgano receptor olfatorio principal y se relaciona con el primer par craneal'15,17. El neuroepitelio olfatorio consta de dos capas: la mucosa olfatoria y la lámina propia. En base al criterio anatómico e inmunohistoquímico, al menos 6 clases de células principales pueden ser identificadas: neuronas sensoriales ciliadas bipolares, células de soporte, células con microvellosidades, células basales globosas, células basales horizontales y células de los ductos de las glándulas submucosas 0 de Bowman ${ }^{17}$. Las neuronas ciliadas bipolares actúan como receptoras y transductoras simultáneamente, mientras que las glándulas de Bowman son capaces de producir el líquido mucoso que disuelve las sustancias odoríferas. Sobre la superficie ciliada existen proteínas de unión específica, que fueron descubiertas por Buck y Axel, y son capaces de captar olores que se han introducido en la película mucosa del epitelio olfatorio ${ }^{15,17}$. Las moléculas odoríferas desencadenan la formación de segundos mensajeros, los que se observan en la cascada de transducción que se activa a nivel distal de los receptores acoplados a las proteínas $\mathrm{G}^{15-17}$.

Las células olfatorias, entre 10 y 15 millones en cada fosa nasal del adulto, se reproducen continuamente por mitosis, y se pierden por descamación. El ciclo dura aproximadamente 3 meses $^{15,17}$. Las células gliales olfatorias forman la vaina de las neuronas olfatorias primarias. Los axones de las neuronas olfatorias se agrupan en cerca de 20 fascículos, que luego formarán los nervios olfatorios que entrarán a la cavidad craneal a través de la lámina cribiforme del etmoides, continuan- 
do hacia el bulbo olfatorio, hacia los llamados glomérulos ${ }^{15,16}$. Las células principales del bulbo olfatorio terminan en áreas olfatorias primarias, para la apreciación subjetiva de los olores, y luego establecer conexión con otras zonas encefálicas para respuestas emocionales y viscerales frente a estímulos ${ }^{15}$. El área olfatoria, llamada corteza piriforme, presente en el lóbulo temporal, cuenta con un centro especializado para el sentido del olfato, y constituye el área de recepción cortical final de la vía olfatoria ${ }^{15,17}$.

La percepción de los aromas resulta de una combinación de la activación del olfato por componentes odoríferos liberados en la nasofaringe, el gusto y sensaciones somatosensoriales como la textura, el calor y el frío, estas últimas mediadas por activación del nervio trigémino ${ }^{15,18}$. Muchos pacientes con disfunción olfatoria refieren disminución en la percepción de sabores. Esto se debe a que la mayoría de los sabores son en realidad olores que ascienden vía retronasal hacia el epitelio olfatorio. Estos pacientes mantienen intacta la capacidad de percibir el gusto salado, dulce, amargo, ácido y umami, funciones que corresponden a las papilas gustatorias, y son mediadas por el nervio facial y glosofarínge ${ }^{18}$.

\section{Patología olfatoria}

La patología olfatoria es una afección bastante común en la población general, puede ser cuantitativa (anosmia/hiposmia) 0 cualitativa (parosmia/fantosmia). Se estima que la anosmia e hiposmia, inhabilidad o habilidad disminuida del olfato, respectivamente, afecta entre $3 \%$ y $20 \%$ de la población mundial ${ }^{19}$, principalmente a adultos mayores; llegando hasta $50 \%$ en los mayores de 65 años, y más de $80 \%$ en los mayores de 80 años, esto puede alterar de forma relevante la calidad de vida de estos pacientes al disminuir la habilidad de reconocer olores de advertencia en alimentos o medioambiente, y también al afectar el área de interacciones sociales, higiene, alimentación, y sensación de bienestar ${ }^{20}$.

\section{Patología cuantitativa}

La anosmia/hiposmia puede ser manifestación de distintas patologías de base, las más frecuentes son enfermedades rinosinusales, trastornos posinfecciosos (infección respiratoria alta), y trastornos postraumáticos, otras etiologías menos frecuentes son causas congénitas, idiopáticas, tóxicos, y enfermedades neurodegenerativas ${ }^{19}$. Otra forma de clasificarlas son etiologías conductivas y/o traumáticas, y etiologías sensorioneurales, descritas en la Tabla 1, aunque algunas etiologías son mixtas, y en varios casos, no se ha logrado determinar la fisiopatología ${ }^{15,20-26}$. Para una revisión de los medicamentos que puedan causar alteración del olfato, referimos al lector al artículo publicado por Susan S. Schiffman ${ }^{27}$.

El consenso general es que el autorreporte del deterioro en el olfato es específico, pero poco sensible, es decir, las personas no reconocen necesariamente el problema, la prevalencia autorreportada varía entre $1,4 \%$ y $15 \%{ }^{28}$. Existen test olfatorios validados que permiten un acercamiento un poco más objetivo al grado de la patología, tales como, el Sniffin' Sticks, el University of Pennsylvania Smell Identification Test (UPSIT), y el test olfatorio del Connecticut Chemosensory Clinical Research Center (CCCRC) ${ }^{28}$. En el estudio endonasal, en general, no se encuentran mayores hallazgos que los relacionados a la patología meramente nasal. El estudio se puede ampliar con imágenes (tomografía computarizada o resonancia magnética) según necesidad; se ha descrito que el volumen del bulbo olfatorio puede usarse para predecir el pronóstico de la disfunción olfatoria ${ }^{20}$.

\section{Anosmia posviral}

La anosmia posinfecciosa es la causa más frecuente de anosmia, hasta en $40 \%$ de los casos. La fisiopatología de base es la congestión primaria de la mucosa, que lleva a obstrucción nasal y pérdida olfativa del tipo obstructiva. En la mayoría de los casos la anosmia resuelve una vez que resuelve el cuadro clínico y la obstrucción, pero algunos pacientes permanecen con anosmia debido a la afección neuronal inducida por el virus $^{29}$. Se cree que uno de los mecanismos sería la disrupción de las neuronas olfatorias ${ }^{20}$. Por otro lado, se ha observado que entre $32 \%$ y $66 \%$ de los pacientes con anosmia posviral tienen recuperación espontánea a 1 y 3 años plazo respectivamente ${ }^{19,28}$. 
Tabla 1. Etiologías de anosmia/hiposmia

\begin{tabular}{|c|c|}
\hline Etiologías conductivas y/o traumáticas & Etiologías sensorioneurales \\
\hline $\begin{array}{l}\text { Enfermedades obstructivas nasales: } \\
\text { - } \quad \text { Pólipos nasals } \\
\text { - } \quad \text { Rinitis alérgica } \\
\text { - } \quad \text { Tumores nasales y de base de cráneo } \\
\text { - } \quad \text { Meningioma, glioma, adenocarcinoma, } \\
\quad \text { estesioneuroblastoma, papiloma invertido } \\
\text { Traumatismo craneal: } \\
\text { - } \quad \text { Contusiones } \\
\text { - } \quad \text { Hematoma } \\
\text { - } \quad \text { Cizallamiento del nervio y/o tracto olfatorio } \\
\text { Posquirúrgica: } \\
\text { - } \quad \text { Traqueostomía } \\
\text { - } \quad \text { Laringectomía total }\end{array}$ & $\begin{array}{l}\text { Posinfección respiratoria alta } \\
\text { Presbiosmia } \\
\text { Congénita: } \\
\text { - } \quad \text { Anosmia congénita aislada } \\
\text { - } \quad \text { Síndrome de Kallman } \\
\text { Enfermedades neurodegenerativas: } \\
\text { - } \quad \text { Enfermedad de Alzheimer } \\
\text { - } \quad \text { Enfermedad de Parkinson } \\
\text { Enfermedades endocrinas: } \\
\text { - } \quad \text { Síndrome de Cushing } \\
\text { - } \quad \text { Hipotiroidismo } \\
\text { - } \quad \text { Pseudohipoparatiroidismo } \\
\text { - } \quad \text { Insuficiencia adrenocortical } \\
\text { Medicamentos } \\
\text { - } \quad \text { Inhibidores de la enzima convertidora de angiotensina } \\
\text { - } \quad \text { Bloqueadores de canales de calcio } \\
\text { - } \quad \text { Estatinas } \\
\text { - } \quad \text { Quimioterapia } \\
\text { Factores ocupacionales y ambientales: } \\
\text { - } \quad \text { Metales (plomo, cadmio, manganeso), } \\
\text { - } \quad \text { Productos químicos (acetona, sulfuro de hidrógeno, } \\
\quad \text { acrilato, metacrilato) } \\
\text { - } \quad \text { Pesticidas (clorpirifós y neonicotinoides) } \\
\text { - } \quad \text { Tabaco }\end{array}$ \\
\hline \multicolumn{2}{|c|}{ Mixta o mecanismo indeterminado } \\
\hline $\begin{array}{l}\text { Rinosinusitis crónica } \\
\text { Posquirúrgica } \\
\text { - } \quad \text { Septoplastía } \\
\text { - } \quad \text { Cirugía endoscópica nasal } \\
\text { Deficiencias nutricionales } \\
\text { Neuropsiquiátricas } \\
\text { Idiopática } \\
\text { Enfermedades crónicas y autoinmune: } \\
\text { - } \quad \text { Miastenia gravis } \\
\text { - } \quad \text { Enfermedad inflamatoria intestinal } \\
\text { - } \quad \text { Enfermedad de Behçet } \\
\text { - } \quad \text { Insuficiencia renal o hepática } \\
\text { - } \quad \text { Diabetes mellitus }\end{array}$ & \\
\hline
\end{tabular}

\section{Anosmia y COVID-19}

Existen múltiples reportes en la literatura de pacientes con anosmia durante una infección con SARS-CoV-2 ${ }^{30-32}$. Los clínicos de distintos servicios de otorrinolaringología han descrito un aumento de hasta nueve veces en las consultas por alteraciones olfativas desde el inicio de la pandemia por COVID-1930,33. Se han propuesto dos posibles mecanismos que podrían resultar en esta alteración del olfato. Podría corresponder a una obstrucción inflamatoria de las hendiduras olfatorias como causa conductiva, o una anosmia posviral con alteración del neuroepitelio olfatorio como causa sensorioneural ${ }^{30}$.

Con la experiencia adquirida con las epidemias de SARS-CoV y MER-CoV, se buscó establecer cuál es el receptor celular para SARS-CoV-2. Se ha demostrado que SARS-CoV-2 utiliza el receptor ACE2, al igual de SARS-CoV, como puerta de entrada celular $^{34}$. La proteína $S$ (spike) de la superficie de los coronavirus permite la unión al receptor 
celular. SARS-CoV-2 requiere también a la serina proteasa TMPRSS2 para la preparación de la proteína $\mathrm{S}$, y subsiguiente fusión de las membranas virales y celulares ${ }^{35}$. Se ha observado que el neuroepitelio olfatorio expresa ACE2 y TMPRSS2 ${ }^{36,37}$. Sin embargo, ni las neuronas sensoriales olfatorias ni las neuronas del bulbo olfatorio expresan estos genes, que sí se expresan en las células de apoyo, las células madre y las células perivasculares ${ }^{38}$. Estos hallazgos sugieren que la infección por SARS-CoV-2 de células no neuronales conducen a la anosmia. Específicamente, se ha propuesto que la pérdida del olfato en los pacientes infectados puede deberse al impacto directo en las células de soporte, las células madre olfativas y las células de Bowman ${ }^{39}$.

Hasta ahora, los reportes de las alteraciones del olfato en pacientes con SARS-CoV-2 han sido variables ya que los métodos ocupados para describir los hallazgos clínicos han sido distintos (Tabla 2). Por ejemplo, Giacomelli y cols evaluaron a pacientes hospitalizados por COVID-19 a través de una entrevista. Describen resultados para 59 pacientes, de los cuales $33,9 \%$ refiere alguna alteración del olfato y/o gusto, mientras que 18,6\% refieren ambas. Ningún paciente relató anosmia únicamente. Lo más frecuente fue anosmia en conjunto con ageusia (5 pacientes). Doce pacientes refieren alteración del olfato y/o gusto previo a la hospitalización. Igualmente, $72,8 \%$ presentó neumonía al hospitalizarse, por lo cual fueron casos más severos, y no se realizó una evaluación objetiva del olfato ${ }^{40}$. Levinson y cols evaluaron a 42 pacientes hospitalizados con síntomas leves, reportando que $35,7 \%$ presentaron anosmia, y $33,3 \%$, disgeusia. Catorce pacientes presentaron anosmia con disgeusia, y solo un paciente presentó anosmia sin disgeusia. En promedio, las alteraciones aparecieron 3,3 días después del inicio de la enfermedad, y duraron 7,1 días (mediana) y 7,6 días (mediana) para la disgeusia y anosmia (rango 7-14 días), respectivamente ${ }^{41}$.

Otro estudio realizado en Francia describe hallazgos de 114 pacientes adultos vistos en el policlínico de infectología u hospitalizados por COVID-19. Describen que $47 \%$ de los pacientes relatan anosmia, y que $85 \%$ de estos pacientes presenta disgeusia. El promedio de duración de la anosmia fue de 8,9 días. Un problema im- portante en este estudio es el seguimiento muy corto, iniciaron el estudio el primero de marzo, y suspendieron el seguimiento el 24 de marzo ${ }^{42}$. Uno de los estudios con mayor tamaño muestral describe los hallazgos de 417 pacientes con síntomas leve a moderados de COVID-19. Fue un estudio multicéntrico con participación de 12 hospitales en Europa. Excluyeron a los pacientes con antecedentes de patología olfativa 0 gustativa previa a COVID-19 y también a los pacientes en cuidados intensivos. Describen que $85,7 \%$ presentó alteración del olfato, principalmente anosmia $(79,6 \%)$, y que la patología olfatoria apareció en conjunto con otros síntomas generales o de índole otorrinolaringológico en 65,4\%; solo apareciendo previo a otros síntomas en 11,8\%. También hubo casos de fantosmia $(12,6 \%)$ y parosmia $(32,4 \%)$. La mayoría de los pacientes recuperaron el olfato dentro de 14 días de la resolución del COVID-19 (72,6\% dentro de los 8 días). Sólo 3,3\% de los casos con hiposmia, y $3,4 \%$ con anosmia, se demoraron más de 15 días. Además, 88\% presentó alteración del gusto. Las alteraciones del olfato y del gusto fueron principalmente estables durante días, pero en un $23,4 \%$, fueron fluctuantes. De los pacientes recuperados con síntomas residuales del olfato 0 del gusto, 53,9\% presentó alteración del olfato aislada, 22,5\% alteración del gusto aislada, y $23,6 \%$ presentó ambas alteraciones. Enfatizan que los pacientes pueden presentar alteración del olfato sin presentar síntomas rinosinusales ${ }^{43}$.

Yan y cols realizaron una encuesta a los pacientes infectados con SARS-CoV-2, la mayoría de Ios cuales no fueron hospitalizados. Evaluaron a 59 pacientes y $22 \%$ presentó anosmia como síntoma inicial. Los pacientes refieren anosmia en 68\% y ageusia en $71 \%$. En el grupo con ageusia, se debe mencionar que dos pacientes presentaron ageusia aguda, pero sin anosmia aguda, ya que presentaban anosmia previa por el antecedente de rinosinusitis crónica. En los pacientes con anosmia, el $72,5 \%$ describe una mejoría en el tiempo, con $56 \%$ presentando mejoría dentro de 14 días, y $18 \%$ antes del mes ${ }^{44}$. En China, realizaron una revisión de casos buscando la incidencia de manifestaciones neurológicas por SARS-CoV-2. Analizaron los datos de 214 pacientes hospitalizados por COVID-19, de los cuales $41,1 \%$ presentó cuadro severo. Refieren hiposmia e hipogeusia en $5,1 \%$ y $5,6 \%$ 
Tabla 2. Prevalencia de alteraciones del olfato y gusto en COVID-19

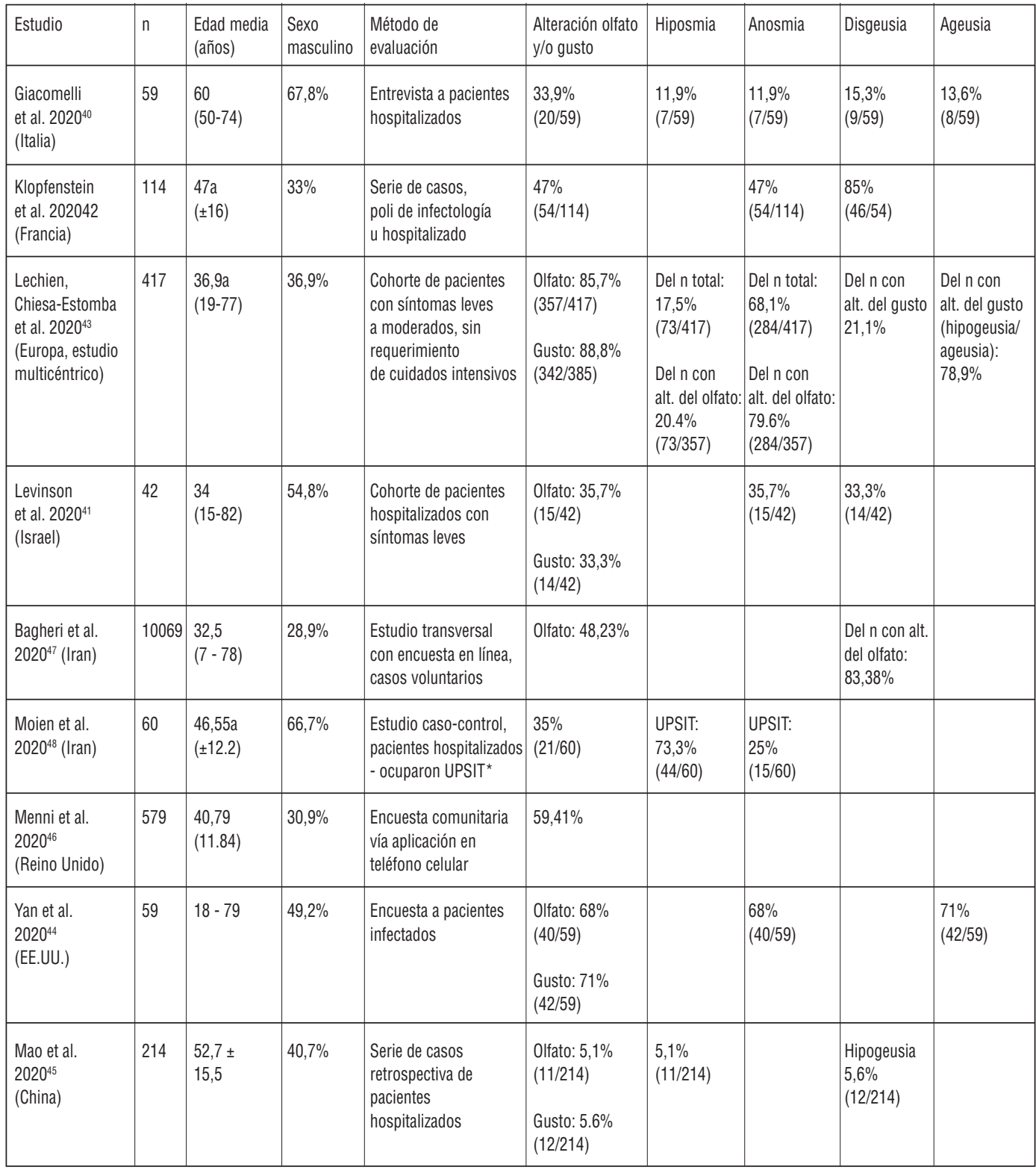

*Prueba de olfato University of Pennsylvania Smell Identification Test (UPSIT). aEdad promedio.

de los pacientes, respectivamente. La mayoría de los casos con hiposmia fueron en los casos no severos $(n=8 / 11)$, lo mismo fue observado para la hipogeusia $(n=9 / 12)^{45}$.

Menni y cols realizaron un estudio con una encuesta comunitaria a toda la población del Reino Unido, que se realizó a través de una aplicación en el teléfono celular llamado COVID RADAR Symptom Tracker app, diseñado para recopilar datos a nivel nacional. Han recopilado datos de más de un millón de habitantes, de los cuales, 1.702 relatan haber realizado la prueba PCR por sus síntomas; 579 fueron positivos. De ellos, 59,41\% relata alteración del olfato y/0 del gusto ${ }^{46}$. Otro estudio 
que también ocupó una plataforma en línea fue Bagheri y cols desarrollaron una encuesta y se les solicitó a los pacientes de forma voluntaria, contestar online si presentaban alguna alteración del olfato dentro de las 4 semanas desde el inicio de la pandemia: 10.069 participantes contestaron la encuesta, 48,23\% presentaron hiposmia/anosmia, y de ellos, 83,38\% presentó alteración del gusto. También describen que la aparición de la anosmia fue aguda en $76,24 \%{ }^{47}$.

De nuestro conocimiento, existe solo un estudio hasta la fecha de la elaboración de este manuscrito, que ha intentado objetivar las alteraciones del olfato debido a COVID-19. Moein y cols realizaron un estudio caso control, con 60 participantes en cada grupo. Los casos fueron hospitalizados y confirmado por PCR. Ocuparon el UPSIT para documentar las alteraciones del olfato describiendo que los pacientes con COVID-19 no solo pueden presentar anosmia (25\%), sino también distintos grados de hiposmia (leve $13 \%$, moderada $27 \%$, severa $33 \%$ ). Otro dato interesante es que no todos los pacientes con alteración del olfato lo reconocen necesariamente, $21 \%$ de los pacientes infectados relatan alguna alteración del olfato o del gusto, mientras que $98 \%$ de los pacientes presentaban alguna alteración en la prueba UPSIT ${ }^{48}$.

\section{Tratamiento}

Si bien aún no existe claridad sobre la fisiopatología de la disfunción olfatoria por COVID-19 y su manejo, sí se han estudiado tratamientos para la hiposmia/ anosmia. En términos generales, se ha descrito el uso de corticoides tanto sistémicos como intranasales, observando eficacia principalmente en las etiologías no conductivas de la hiposmia/ anosmia. En el caso de los corticoides sistémicos se ha demostrado su utilidad en pacientes con rinosinusitis crónica de base ${ }^{20}$, pero tanto la dosis como la duración del tratamiento muestra gran variación y se mantiene controversial ${ }^{19}$. Respecto a los corticoides intranasales en suspensión para pulverización nasal o en gotas, mejorarían la función olfatoria en los pacientes con rinitis alérgica ${ }^{19}$ y en algunos casos de hiposmia/anosmia posviral20, siendo importante la adecuada administración de éstos, mejorando su llegada al posicionar la cabeza del paciente reclinada hacia adelante y abajo $0^{49}$.
Sin embargo, en contexto de la actual pandemia por SARS-CoV-2, Lavinsky y cols recomiendan evitar el uso de la terapia corticoidal sistémica en pacientes con síntomas sugerentes de COVID-19, y mantener el uso de corticoides intranasales sólo en los pacientes que los usaban de forma crónica por indicación médica ${ }^{10}$.

Por otro lado, el zinc ha sido uno de los tratamientos más citados y controversiales en la literatura médica para alteraciones olfatorias y gustativas. Varios estudios han fallado en demostrarlo como una terapia efectiva para los trastornos generales del olfato y del gusto ${ }^{49}$, por lo que su uso ha disminuido en el último tiempo. Además, en las dosis prescritas para estos trastornos, algunos pacientes podrían verse afectados por efectos adversos, tales como, déficit de hierro, déficit de cobre, neutropenia, y función inmune alterada ${ }^{49}$.

Recientemente, se han realizado estudios sobre el potencial de la rehabilitación olfatoria. Los estudios se hicieron en pacientes con hiposmia/ anosmia identificada por el test de Sniffin' Sticks. Se comparó la variación de scores pre y posentrenamiento olfatorio. Algunos estudios incluyeron pacientes únicamente con alteraciones olfatorias posvirales ${ }^{50}$, otros, tanto a pacientes con etiología posvirales como postraumáticas ${ }^{51}$, y otros, solo con etiología postraumática ${ }^{52}$. Se han descrito el uso de bolas de entrenamiento olfatorio ${ }^{50}$, y de sets de tubos de 4 olores de entrenamiento (rosa, eucalipto, limón, y clavos de olor $)^{51,52}$, los que deben olerse por 5 minutos, 2 veces al día, al menos por 12 semanas (entre 12 y 24 o más semanas). Konstantinidis y cols reportaron un aumento en los scores de $67,8 \%$ y $33,2 \%$, en los casos posvirales y postraumáticos, respectivamente, luego de 16 semanas de entrenamiento olfatorio ${ }^{51}$. Saatci y cols reportaron que, a las 12 semanas de tratamiento, los scores de los test fueron significativamente mayores en los pacientes entrenados con las bolas de entrenamiento olfatorio versus los con tubos de 4 olores de entrenamiento ${ }^{50}$. Pellegrino y cols, reportaron mejoría en los scores de los test respecto a los basales, y cambios en el volumen del bulbo olfatorio evaluados por resonancia magnética. Los pacientes estuvieron en rehabilitación olfatoria por 240 más semanas, con los sets de 4 olores $^{52}$. Para mayor detalle de los distintos tratamientos estudiados en alteracio- 
nes olfatorias y gustativas, referimos al lector la revisión de Richard L. Doty ${ }^{49}$.

\section{CONCLUSIÓN}

Luego de una revisión exhaustiva de la evidencia publicada a la fecha, podemos concluir que en contexto de la pandemia por COVID-19 han aumentado considerablemente las consultas por alteraciones en el olfato, muchas veces sin otros síntomas acompañantes al momento de la atención clínica pero que podría tratarse de pacientes portadores de SARS-CoV-2, situación que expone a mayor riesgo al personal de salud, especialmente al otorrinolaringólogo, que generalmente es el especialista al que consultan este tipo de pacientes, por lo tanto, se hace indispensable en estos tiempos mantener las precauciones estándar de higiene y el uso de elementos de protección personal atingentes a la hora del enfrentamiento clínico. Más importante aún, es buscar evidencia que permita decidir si la detección precoz de alteraciones del olfato sería un posible método de screening de COVID-19, pudiendo indicar medidas de aislamiento precoz a pacientes oligosintomáticos disminuyendo así la posibilidad de infectar a otros.

Por otro lado, dada la variabilidad en la metodología utilizada en los distintos estudios para describir los hallazgos clínicos de alteraciones del olfato en pacientes con SARS-CoV-2, no se puede concluir con un grado de certeza fuerte la asociación directa entre la patología olfatoria y gustativa con COVID-19, pero resulta evidentemente llamativo el número de reportes y resultados de los estudios existentes hasta la fecha, pudiendo existir alguna relación pero aún no sabemos la magnitud de ésta, por lo que es un desafío continuar con investigaciones en el área, comenzando por incluir el déficit olfatorio y/o gustativo dentro de la anamnesis estándar ante un caso sospechoso de SARS-CoV-2. Además, surge la necesidad de contar con material estandarizado para realizar el cuestionario a los casos de COVID-19 ya sea en su estadía hospitalaria 0 a distancia. En este sentido, surge como una buena alternativa el test de UPSIT que, por su fácil aplicación, sin necesidad de presencia de personal calificado y que posterior a su realización se elimina, pareciera ser la herramienta ideal para cuantificar el olfato en estos pacientes, considerando además su amplio uso y vali $\neq$ dación en diferentes países del mundo.

La anosmia/hiposmia implica para muchos pacientes un mayor riesgo en su vida diaria, pues el medio en el que nos desenvolvemos está lleno de olores que colaboran a nuestra seguridad, esto se hace evidente cuando percibimos el aroma a gas 0 el olor del humo, situaciones que deberían alarmarnos que algo más está sucediendo, pero en el caso de los individuos que sufren alteraciones olfatorias, no logran notar estas señales, por lo que podrían ser víctimas de intoxicaciones por gases e incluso ingerir alimentos en mal estado al no darse cuenta por medio del sentido del olfato que el producto pudiese estar descompuesto. Es de suma importancia explicarles a los pacientes con anosmia estos riesgos y, además, sugerir el uso de detectores de gas y monóxido de carbono con alarmas. En la misma línea de lo alimentario, sabemos que existe relación entre el gusto y el olfato, y que generalmente estas alteraciones se acompañan; pudiendo verse comprometida la nutrición del paciente al no percibir los sabores de los alimentos, llevando a una conducta restrictiva, es decir, disminución del apetito al no disfrutar del sabor de la comida, o por el otro lado, a una conducta que no sea favorable para su salud al sobresazonar los alimentos, esto toma aún mayor peso en los pacientes con diabetes y/o hipertensión arterial.

Por lo anterior, el otorrinolaringólogo juega un rol importante en beneficio de los pacientes que sufran alteraciones olfatorias $\mathrm{y} / 0$ gustativas, al indicar algún tipo de manejo, los estudios muestran promisorios resultados respecto a la rehabilitación olfatoria. 


\section{BIBLIOGRAFÍA}

1. Schoeman D, Fielding BC. Coronavirus envelope protein: current knowledge. Virol J 2019; 16. doi:10.1186/s12985-019-1182-0.

2. De Wit E, van Doremalen N, Falzarano D, Munster V. SARS and MERS: recent insights into emerging coronaviruses. Nat Rev Microbiol 2016; 14 : 523-34. doi:10.1038/nrmicro.2016.81.

3. World Health Organization. Coronavirus disease 2019 (COVID-19) Situation Report - 97. WHO [Internet]. 2020. [cited 2020 April 26]. Available from: https://www.who.int/emergencies/diseases/ novel-coronavirus-2019/situation-reports/.

4. Ministerio de Salud de Chile. Casos confirmados en Chile COVID-19. MINSAL [Internet]. 2020. [cited 2020 April 26]. Available from: https:// www.minsal.cl/nuevo-coronavirus-2019-ncov/ casos-confirmados-en-chile-covid-19/.

5. Asadi S, Bouvier N, Wexler AS, Ristenpart WD. The coronavirus pandemic and aerosols: Does COVID-19 transmit via expiratory particles? Aerosol Science and Technology 2020; 54: 6358. doi:10.1080/02786826.2020.1749229.

6. Van Doremalen N, Bushmaker T, Morris DH. Aerosol and Surface Stability of SARS-CoV-2 as Compared with SARS-CoV-1. N Engl J Med 2020; 382: 1564-156. doi:10.1056/NEJMc2004973.

7. HeX, Lau EHY, Wu P, et al. Temporal dynamics in viral shedding and transmissibility of COVID-19. Nat Med 2020. doi:10.1038/s41591-020-0869-5.

8. Tindale L, Coombe M, Stockdale JE, et al. Transmission interval estimates suggest pre -symptomatic spread of COVID-19. MedRxiv 2020. doi:10.1101/2020.03.03.20029983.

9. SIORDIA J. Epidemiology and clinical features of COVID-19: A review of current literature. Journal of Clinical Virology 2020; 127. doi:10.1016/j. jcv.2020.104357.

10. Lavinsky J, Kosugi eM, Baptistella E, et al. An update on COVID-19 for the otorhinolaryngologist - a Brazilian Association of Otolaryngology and Cervicofacial Surgery (ABORL-CCF) Position Statement. Braz J Otorhinolaryngol 2020; 885: 1-8. doi:10.1016/j.bjorl.2020.04.002.

11. Krajewska J, Krajewski W, Zub K, Zatonski T. COVID-19 in otolaryngologist practice: a review of current knowledge. Eur Arch Otorhinolaryngol 2020. doi:10.1007/s00405-020-05968-y.
12. Lovato A, de Filippis A, Marioni G. Upper airway symptoms in coronavirus disease 2019 (COVID-19). American J Otolaryngol-Head and Neck Medicine and Surgery 2020. doi:10.1016/j.amjot0.2020.102474.

13. Wu Z, McGoogan JM. Characteristics of and Important Lessons from the Coronavirus Disease 2019 (COVID-19) Outbreak in China: Summary of a Report of 72314 Cases from the Chinese Center for Disease Control and Prevention. JAMA [Internet]. 2020 [cited 2020 April 26]. Available from: https://jamanetwork.com/journals/jama/ fullarticle/2762130.

14. Hopkins C, Kumar N. Loss of sense of smell as marker of COVID-19 infection. ENT UK [Internet]. 2020 [cited 2020 April 26]. Available from: https://www.entuk.org/sites/default/files/files/ Loss $\% 20$ of $\% 20$ sense $\% 20$ of $\% 20$ smell $\% 20$ as\%20marker\%20of\%20COVID.pdf.

15. Carrillo B, Carrillo V, Astorga A, Hormachea D. Diagnóstico en la patología del olfato: Revisión de la literatura. Rev Otorrinolaringol Cir Cabeza Cuello 2017; 77: 351-60. doi:10.4067/s071848162017000300351.

16. Sмith T, Bhatnagar K. Chapter 2 - Anatomy of the Olfactory System. Handbook of Clinical Neurology 2019; 164: 17-28. doi:10.1016/B9780-444-63855-7.00002-2.

17. Fuentes A, Fresno MJ, Santander H, Valenzuela S, GutiérRez MF, Millares R. Sensopercepción olfatoria: una revisión. Rev Med Chile 2011; 139: 362-7.

18. Machado C, Gutierrez J. Anosmia and Ageusia as Initial or Unique Symptoms after SARS-COV-2 Virus Infection. Preprints 2020. doi:10.20944/ preprints202004.0272.v1.

19. Boesveldt S, Postma E M, Boak D, et al. Anosmia - A Clinical Review. Chemical Senses 2017; 42: 513-23. doi: 10.1093/chemse/bjx025.

20. Scangas GA, Bleier BS. Anosmia: Differential Diagnosis, Evaluation, and Management. $\mathrm{Am} \mathrm{J}$ Rhinol Allergy 2017; 31(1): 3-7. doi: 10.2500/ ajra.2017.31.4403.

21. Leon-Sarmiento FE, Bayona Ea, Bayona-Prieto J, Osman A, Doty RL. Profound olfactory dysfunction in myasthenia gravis. PLOS One 2012; 7(10): e45544. doi:10.1371/journal. pone. 0045544 .

22. Steinbach S, Reindl W, Dempfle A, et al. Smell and taste in inflammatory bowel disease. PLOS 
One 2013; 8(9): e73454. doi:10.1371/journal. pone.0073454.

23. Akyol L, Günbey E, Karli R, Önem S, Özgen M, SaYarLIOGLU M. Evaluation of olfactory function in Behçet's disease. Eur J Rheumatol 2016; 3(4): 153-6. doi:10.5152/eurjrheum.2016.017.

24. Lee SJ, Kim EM, Cho SH, Song J, Jang TW, Lee MY. Risk of olfactory dysfunction of the workers in the automobile repair, printing, shoemaking and plating industries in Korea: a cross-sectional study. BMJ Open 2018; 8: e022678. doi:10.1136/ bmjopen-2018-022678.

25. Werner S, Nies E. Olfactory dysfunction revisited: a reappraisal of work-related olfactory dysfunction caused by chemicals. J Occup Med Toxicol 2018; 13: 28. doi:10.1186/s12995-018-0209-6.

26. Daramola 00, Becker SS. An algorithmic approach to the evaluation and treatment of olfactory disorders. Curr Opin Otolaryngol Head Neck Surg 2015; 23: 8-14. doi:10.1097/ M00.0000000000000118.

27. Schiffman S. Influence of medications on taste and smell. World Journal Otorhinolaryngol-Head and Neck Surgery 2018; 4: 84-91. doi:10.1016/j. wjorl.2018.02.005.

28. Walliczek-Dworschak U, Hummel T. The Human Sense of Olfaction. Facial Plast Surg 2017; 33: 396-404. doi:10.1055/s-0037-1603828.

29. Galougahi MK, Ghorbani J, Bakhshayeshikaram M, NaeinI AS, Hasel S. Olfactory Bulb Magnetic Resonance Imaging in SARS-CoV-2-Induced Anosmia: The First Report. Academic Radiology 2020. doi:10.1016/j.acra.2020.04.002.

30. Gane S, Kelly C, Hopkins C. Isolated sudden onset anosmia in COVID-19 infection. A novel syndrome? Rhinology 2020; 58. doi:10.4193/ Rhin20.114.

31. Villalba L, Maouche Y, Alonso M B, et al. Anosmia and dysgeusia in the absence of other respiratory disease: should COVID-19 infection be considered? EJCRIM 2020; 7. doi:10.12890/2020 001641.

32. Ollarves-Carrero M, Rodriguez-Morales A, BonillaAldana DK, Rodriguez-Morales AJ. Anosmia in a healthcare worker with COVID-19 in Madrid, Spain. Travel Medicine and Infectious Disease 2020. doi:10.1016/j.tmaid.2020.101666.

33. Xydakis M, Dehgani-Mobaraki P, Holbrook E H, et AL. Smell and taste dysfunction in patients with
COVID-19. Lancet Infect Dis 2020. doi:10.1016/ S1473-3099(20)30293-0.

34. Zhou P, Yang $X$, Wang $X$, et al. A pneumonia outbreak associated with a new coronavirus of probable bat origin. Nature 2020; 579: 270-3. doi:10.1038/s41586-020-2012-7.

35. Hoffmann M, Kleine-Weber H, Schroeder S, et al. SARS-CoV-2 Cell Entry Depends on ACE2 and TMPRSS2 and Is Blocked by a Clinically Proven Protease Inhibitor. Cell 2020; 181: 271-80. doi:10.1016/j.cell.2020.02.052.

36. Butowt R, BILINSKA K. SARS-CoV-2: Olfaction, Brain Infection, and the Urgent Need for Clinical Samples Allowing Earlier Virus Detection. ACS Chem Neurosci 2020. doi:10.1021/ acschemneuro.0c00172.

37. Fodoulian L, Tuberosa J, Rossier D, Landis BN, Carleton A, Rodriguez I. SARS-CoV-2 receptor and entry genes are expressed by sustentacular cells in the human olfactory neuroepithelium. BioRxiv2020. doi:10.1101/2020.03.31.013268.

38. Brann DH, Tsukahara T, Weinreb C, et al. Nonneuronal expression of SARS-CoV-2 entry genes in the olfactory system suggests mechanisms underlying COVID-19-associated anosmia. BioRxiv2020. doi:10.1101/2020.03.25.009084.

39. Gupta K, Mohanty SK, KalRa S, et al. The molecular basis of loss of smell in 2019-nCoV infected individuals. Preprints 2020. doi:10.21203/ rs.3.rs-19884/v1.

40. Giacomelli A, Pezzati L, Conti F, et al. Self-reported olfactory and taste disorders in SARS-CoV-2 patients: a cross-sectional study. Clinical Infectious Diseases 2020. doi:10.1093/cid/ ciaa330.

41. Levinson R, Elbaz M, Ben-Amı R, et al. Anosmia and dysgeusia in patients with mild SARS-CoV-2 infection. MedRxiv 2020. doi:10.1101/2020.04. 11.20055483.

42. Klopfenstein T, Kadiane-Oussou NJ, Toko L, et al. Features of anosmia in COVID-19. Medecine et Maladies Infectieuses 2020, doi:10.1016/j. medmal.2020.04.006.

43. Lechien J, Chiesa-Estomba C, De Siati D, et al. Olfactory and gustatory dysfunctions as a clinical presentation of mild-to-moderate forms of the coronavirus disease (COVID-19): a multicenter European study. Eur Arch Otorhinolaryngol 2020. doi:10.1007/s00405-020-05965-1. 
44. Yan C, Farajl F, Prajapati D, Boone C, DeConde A. Covid-19 Associated Smell and Taste Loss 2020. doi:10.1111/alr.22579.

45. Mao L, Wang M, Chen S, et al. Neurological Manifestations of Hospitalized Patients with COVID-19 in Wuhan, China: a retrospective case series study. MedRxiv 2020. doi:10.1101/2020 02.22.20026500.

46. Menni C, Valdes AM, Freidin MB, et al. Loss of smell and taste in combination with other symptoms is a strong predictor of COVID-19 infection. MedRxiv 2020. doi:10.1101/2020.04. 05.20048421.

47. Bagheri SH, Asghari A, Farhadi M, et al. Coincidence of COVID-19 epidemic and olfactory dysfunction outbreak. MedRxiv 2020. doi:10.1101/2020.03. 23.20041889.

48. Moein ST, Hashemian SMR, Mansourafshar B, Khorram-Tousi A, Tabarsi P, Doty RL. Smell Dysfunction: A Biomarker for COVID-19. Int
Forum Allergy Rhinol 2020. doi:10.1002/ alr.22587.

49. Doty RL. Treatments for smell and taste disorders: A critical review. Handbook of Clinical Neurology 2019; 164: 455-79. doi:10.1016/ B978-0-444-63855-7.00025-3.

50. Saatci 0, Altundag A, Duz OA, Hummel T. Olfactory training ball improves adherence and olfactory outcomes in post-infectious olfactory dysfunction. Eur Arch Otorhinolaryngol 2020. doi:10.1007/s00405-020-05939-3.

51. Konstantinidis I, Tsakiropoulou E, Bekiaridou P, Kazantzidou C, Constantinidis J. Use of Olfactory Training in Post-Traumatic and Postinfectious Olfactory Dysfunction. The Laryngoscope 2013. doi:10.1002/lary.24390.

52. Pellegrino R, Han P, Reither N, Hummel T. Effectiveness of Olfactory Training on Different Severities of Posttraumatic Loss of Smell. The Laryngoscope 2019. doi:10.1002/lary.27832. 